\title{
Miami combined ILLRS LLRS and ASAMI-BR Conference Presentations by Dr. M M Bari
}

\section{Bari MM*}

Chief consultant, Bari-Ilizarov Orthopaedic Centre, Visiting and Honored Professor of ilizarov center Kurgan Russia

Submission: November 20, 2015; Published: December 09, 2015

*Corresponding author: Md Mofakhkharul Bari, Bari-Ilizarov Orthopaedic Centre, 72, Satmasjid Road, Nizams Shankar Plaza, Dhanmondi, Dhaka, Bangladesh, Tel: +8801819211595; Email: bari.ilizarov31@gmail.com

\section{\#8: Limb Reconstruction Procedures in Tibial Hemimelia}

Question: How to treat- 1 . Reconstruct weight bearing bone with controlled knee bone function 2. To equalize the LLD 3. To correct any ankle and foot deformity and make the foot plantigrade.

Methods: 12 cases of different tibial hemimelia, 8 boys and 4 girls were treated from 1995 to 2013 at NITOR and Bari-Ilizarov Orthopaedic Centre. In 9 cases the right side was affected, the left side in 2 cases and bilateral affection in 1 case. The procedure that was used is the surgical technique (Brown procedure) to centralize the fibula at the knee and ankle with stabilization by Ilizarov technique. Sometimes reconstruction of knee extensor mechanism and deficient ligament reconstruction in ankle is needed. Ilizarov technique is absolutely needed to correct the knee, ankle and foot deformities and to overcome the LLD.

Results: The results that we obtained were very much satisfactory in all cases. Complications are a fact of life that every orthopaedic surgeon has to face. In my series 1 refracture was observed and that was treated by reapplication of Ilizarov and union was achieved.

Conclusion: Ilizarov technique is a reliable and adaptable technique by which we can correct deformity and gain limb length simultaneously by intelligent meticulous follow-up. 


\section{\#9: Fibular Hemimelia}

Question: How to treat- 1. Reconstruct weight bearing bone with controlled knee bone function 2. To equalize the LLD 3. To correct any ankle and foot deformity and make the foot plantigrade.

Methods: 18 patients (12 males and 6 females) treated because of type II Aichterman -Kalamchi fibular hemimelia. 12 segments were lengthened ( 8 tibia, 4 femur). The tibia was lengthened twice in six patients, simultaneous tibia and femoral lengthening in 4 cases. The age at surgery ranged from 2-15 years (mean 8 years); mean shortening was $7.5 \mathrm{~cm}$ (ranged from 3-15 cm). Valgus deviation in 12 patients, in 2 of them combined with rotational deformity. llizarov frame used for tibial lengthening in 10 segments. Hind foot stabilization was done in all the patients. Femoral lengthening was done in 4 patients.

Results: Lengthening achieved from 3-9 cm. Axial deviation (valguS, detorsion 4) was corrected in 8 patients.

Conclusion: Treatment of fibular hemimelia is very difficult, particularly lengthening and it is technically demanding, required multiple surgeries and entails a lengthy procedure with many complications anticipated. 


\section{5: Cosmetic Orthopaedics- by Ilizarov Technique}

Question: How to correct the congenital, acquired and posttraumatic deformities?

Methods: All the deformities can be corrected by Ilizarov technique. Surgery for anthropomettic corrections to correct the form of extremities is Cosmetic Orthopaedics. Cosmetic orthopaedics is that branch of medical science that making a person beautiful though changing his limb form, improvement in the appearance of physical feature and defects. llizarov is the most natural way of treating all bones and joint problems. Results: Results are excellent and good.

Conclusion: All the deformities with shortening can be corrected simultaneously by llizarov technique. 


\section{\# 137: Correction of Fasciogenitopopliteal Syndrome by Ilizarov External Fixator}

Question: Popliteal pterygium syndrome is rare congenital anomaly characterized by a web like skin formation in the Popliteal area leading to fixed flexion contracture with an incidence of less than 1 in 300000 . Most of the literature is on its clinical description rather than on its treatment. After Trelat's initial description in 1869, Gorlin was the first to introduce the term Popliteal Pterygium Syndrome (PPS) in 1968. It has been associated with other congenital anomalies and is further known as "faciogenitopopliteal syndrome". This may be due to Dermatogenic, Desmogenic, Myogenic, Osteogenic, Neurogenic, Primary treatment goals are improvement in functional extension, hygiene independence and cosmesis. Reported treatment options are anterior femoral stapling, supracondylar extension osteotomy, femoral shortening and Ilizarov external fixator.

Methods: We report 8 cases with severe webbing causing FFD of both knee joints (ages ranged from 3-14 years). One 14 years old boy presented to us with severe right sided webbing causing FFD of knee with $8 \mathrm{~cm}$ shortening ( $3 \mathrm{~cm}$ femur, $5 \mathrm{~cm}$ tibia fibula), his daily activities were limited a movement was only by crawling or jumping. He used to walk upright. We corrected his deformity using gradual soft tissue distraction with Ilizarov fixator for 120 days. After correction of the deformity, we lengthened his tibia fibula; immediately after removal of Ilizarov fixator he was in plaster for four weeks.

Conclusion: Correcting Popliteal pterygium syndrome with Ilizarov method is a good choice in managing this difficult problem.

ILIZAROV means: = You must have intelligent

L: You can lengthen limb whatever you like; I: You can control infection; Z: You can correct Zigzag (deformity); A: You will get angiogenesis with adaptation; R: This technique is of course reliable with a lot of; Opportunities, having; V: Versatile effect. 


\section{\#157: Judet's Quadricepsplasty for Knee Contracture}

Question: Is Judet's Quadricepsplasty applicable for correction $\mathrm{o}^{\prime}$ the knee extension contracture?

Methods: Yes, it is corrected by Judet's Quadricepsplasr. Results: Results are uniformly unique.

Conclusion: By doing 5 steps surgery we can correct up to 9: degree or more flexion of knee extension contracture. It is a useful procedure to increase the range of motion of rigid knees. 


\section{\#229: 1lizarov Applications to Benign Bone Tumors}

Question: Is there any usefulness of llizarov external fixator for the treatment of benign bone tumors?

Methods: We treated 29 limbs of 27 patients with deformity and different LLD due to benign bone tumor. There were 20 males and 7 female with a mean age of 11 years. We used Ilizarov of different bone tumor. The etiologies were osteochondroma in 9 patients. Olliers disease in 5 patients, fibrous dysplasia in 8 patients and GCT in 5 patients.

Results: The outcomes of the results were satisfactory in case of all these benign bone tumors.

Conclusion: Preservation and bone degeneration by means of distraction osteogenesis constitutes a highly conservative limb saving surgery. Patients with good defects of less than $10 \mathrm{~cm}$, a great deal of preserve healthy tissue and good prognosis are good candidates for these methods. 